\title{
Gas Sloshing Regulates and Records the Evolution of the Fornax Cluster
}

\author{
Yuanyuan $\mathrm{Su}^{1}$, Paul E. J. Nulsen ${ }^{1}$ (1) , Ralph P. Kraft ${ }^{1}$ (10, Elke Roediger $^{2}$, John A. ZuHone ${ }^{1}$, Christine Jones ${ }^{1}$, William R. Forman ${ }^{1}$ (1), \\ Alex Sheardown ${ }^{2}$, Jimmy A. Irwin ${ }^{3}$ (D), and Scott W. Randall ${ }^{1}$ (D) \\ ${ }^{1}$ Harvard-Smithsonian Center for Astrophysics, 60 Garden Street, Cambridge, MA 02138, USA; yuanyuan.su@cfa.harvard.edu \\ ${ }^{2}$ E.A. Milne Centre for Astrophysics, School of Mathematics and Physical Sciences, University of Hull, Hull, HU6 7RX, UK \\ ${ }^{3}$ Department of Physics and Astronomy, University of Alabama, Box 870324, Tuscaloosa, AL 35487, USA \\ Received 2017 August 5; revised 2017 October 18; accepted 2017 November 4; published 2017 December 13
}

\begin{abstract}
We present results of a joint Chandra and XMM-Newton analysis of the Fornax Cluster, the nearest galaxy cluster in the southern sky. Signatures of merger-induced gas sloshing can be seen in the X-ray image. We identify four sloshing cold fronts in the intracluster medium, residing at radii of $3 \mathrm{kpc}$ (west), $10 \mathrm{kpc}$ (northeast), $30 \mathrm{kpc}$ (southwest), and $200 \mathrm{kpc}$ (east). Despite spanning over two orders of magnitude in radius, all four cold fronts fall onto the same spiral pattern that wraps around the BCG NGC 1399, likely all initiated by the infall of NGC 1404. The most evident front is to the northeast, $10 \mathrm{kpc}$ from the cluster center, which separates low-entropy highmetallicity gas and high-entropy low-metallicity gas. The metallicity map suggests that gas sloshing, rather than an AGN outburst, is the driving force behind the redistribution of the enriched gas in this cluster. The innermost cold front resides within the radius of the strong cool core. The sloshing timescale within the cooling radius, calculated from the Brunt-Väsälä frequency, is an order of magnitude shorter than the cooling time. It is plausible that gas sloshing is contributing to the heating of the cool core, provided that gas of different entropies can be mixed effectively via Kelvin-Helmholtz instability. The estimated age of the outermost front suggests that this is not the first infall of NGC 1404.
\end{abstract}

Key words: galaxies: clusters: intracluster medium - X-rays: galaxies: clusters

\section{Introduction}

A long-lasting puzzle in extragalactic astronomy is why galaxy clusters come in two varieties: cool-core clusters and non-cool-core clusters (Jones \& Forman 1984). The former features a sharp X-ray emission peak, owing to the radiative cooling of a dense, cool, and enriched core. Gas in the cool core emits strongly in X-rays with a radiative cooling time much shorter than the age of the cluster. If there is no heating to compensate for radiative losses, a cooling flow with prodigious star formation is expected. High-resolution X-ray observations from Chandra and XMM-Newton have, however, revealed little low-temperature gas, posing the so-called "cooling problem" (see Peterson \& Fabian 2006 for a review). Feedback from active galactic nuclei (AGNs) may have provided the heat required to balance radiative cooling, despite the fact that the interplay between cooling and feedback remains a subject of debate. In contrast, the gaseous, thermal, and chemical distributions of non-cool-core clusters are relatively homogenous (Cavagnolo et al. 2009; Sanderson et al. 2009).

A phenomenon called the "sloshing cold front" seems to be exclusively associated with cool-core clusters. Sloshing cold fronts produce characteristic spiral or bow-like features in the X-ray surface brightness in cluster centers (Markevitch \& Vikhlinin 2007). Gas sloshing can be triggered by minor mergers or off-axis mergers that disturb the gas at the bottom of a cluster potential. The steep central entropy gradient in a coolcore cluster allows sloshing to bring low-entropy central gas into contact with the higher entropy intracluster medium (ICM), creating an abrupt step in the X-ray emissivity that appears as a "sloshing cold front"; this speculation has been confirmed by simulation (Ascasibar \& Markevitch 2006).

Whether sloshing can contribute to the suppression of cooling depends on the microscopic physics in the ICM-how effectively heat can be transported. Cold fronts are shear interfaces, i.e., the two layers of gas move with respect to each other parallel to the interface. In a purely hydrodynamical context, this configuration inevitably leads to the KelvinHelmholtz instability (KHI; Chandrasekhar 1961; Lamb 1932), which promotes turbulent mixing between gas of different phases. KHI are often identified as "roll" features just outside the interface in simulations and can, in principle, be observed directly as fine structures at the interface. Deviations from pure hydro, in particular viscosity and magnetic fields, can suppress $\mathrm{KHI}$ and preserve the sharpness of the cold front (ZuHone et al. 2011; Roediger et al. 2013). A growing number of deep Chandra observations favor an inviscid ICM based on the presence of KHI eddies (Ichinohe et al. 2017; Su et al. 2017a). Vikhlinin et al. (2001) demonstrate that it requires an ordered magnetic field of $10 \mu \mathrm{G}$ to suppress instability. This value is $10 \times$ larger than the strength of the intracluster magnetic field inferred from Faraday rotation and inverse Compton (Govoni \& Feretti 2004). It was suggested that a weak, tangled magnetic field can be stretched by shear in the sloshing gas to form a magnetic layer parallel to the cold front (Keshet et al. 2010). The formation of such a field structure, called "magnetic draping," can protect the front by inhibiting the growth of KHI (Lyutikov 2006; Dursi \& Pfrommer 2008). This process has been reproduced in magnetohydrodynamics (MHD) simulations (ZuHone et al. 2011). If high- and low-entropy gas on either side of the interface cannot be effectively mixed, then the heating of the core due to sloshing would be modest.

The atmospheres of galaxy clusters are observed to have elemental abundances that are approximately one-third of the solar value $\left(Z_{\odot}\right)$ over the bulk of their volume $(0.3-1.0$ virial radius), which may have been enriched early on by supernovae and/or by the accumulation of the metals stripped from infalling galaxies (e.g., Dupke \& White 2000; De Grandi \& Molendi 2001). Cluster centers ( $r<0.3$ virial radius) often 
show an enhanced metallicity of approximately $Z_{\odot}$. The excess metals are believed to be stellar ejecta from the brightest cluster galaxy (BCG). However, the extent of the $\simeq Z_{\odot}$ gas is often greater than the optical extent of the BCG, causing a positive gradient in the iron-mass-to-light ratio (e.g., David \& Nulsen 2008). This gradient implies that some mechanism transports enriched central gas outward (Rebusco et al. 2005). A number of observations of high-metallicity low-entropy gas surrounding buoyantly rising AGN bubbles suggest that AGN outbursts are capable of displacing gas from the cluster center (e.g., Nulsen et al. 2002; Simionescu et al. 2009; Kirkpatrick \& McNamara 2015). On the other hand, gas motion induced by mergers is also considered to be a mechanism to stir up the central gas. In cool-core clusters, spatial correlations are found between the sloshing front and the elevated metallicities (Blanton et al. 2011; Ghizzardi et al. 2014; Sanders et al. 2016b), demonstrating that gas sloshing is capable of lifting a significant amount of metals from the cluster center. More generally, the role of mergers in redistributing enriched gas is manifested in an apparent association between a more disturbed cluster atmosphere and a more extended metallicity distribution (De Grandi \& Molendi 2001; Rossetti \& Molendi 2010; Su et al. 2016).

In this paper, we present a case study of the nearest cluster in the southern sky, the Fornax Cluster $\left(D_{L}=19 \mathrm{Mpc}\right.$, $1^{\prime}=5.49 \mathrm{kpc}, z=0.00475$, Su et al. 2017a, 2017c), which is a low-mass cool-core cluster. We show that the Fornax Cluster displays all the characteristics of a sloshing cluster, such as a spiral-shaped asymmetry (alternating pattern) in X-ray brightness, temperature, and metallicity. The BCG of this cluster, NGC 1399, harbors a pair of symmetric radio lobes coincident with two X-ray cavities along a north-south axis. Their properties are presented in a separate publication ( $\mathrm{Su}$ et al. 2017b; hereafter Paper I). Its cooling radius as a weak cool core (with a cooling time below $7.7 \mathrm{Gyr}$ ) is $25 \mathrm{kpc}$, while as a strong cool core (with a cooling time below $1 \mathrm{Gyr}$ ) it is $4.5 \mathrm{kpc}$. We found in NGC 1399 that cool gas uplifted by AGN bubbles can account for all of the gas that is expected to cool catastrophically, although the uplifted cool gas may eventually fall back. Its second brightest galaxy, NGC 1404, is falling inward through the ICM from the southeast and features a sharp merging cold front and a stripped gaseous tail, which have been studied in detail in Machacek et al. (2005) and Su et al. (2017a, 2017c). The large-scale ICM has been observed extensively in the X-ray. ROSAT observations indicate an average ICM temperature of $<1.5 \mathrm{keV}$ (Rangarajan et al. 1995; Jones et al. 1997; Paolillo et al. 2002). A mosaic Chandra observation with ten $50 \mathrm{ks}$ pointings reveals an asymmetry in its morphology and temperature structures (Scharf et al. 2005). Using joint Suzaku and XMM-Newton mosaic observations, Murakami et al. (2011) find an average metallicity of near the solar value in the cluster center that declines to $\approx 0.3 Z_{\odot}$ at large radii, similar to that of many other galaxy clusters and groups. This study focuses on the effect of gas sloshing in the ICM. The observations and data reductions are described in Section 2. Further analysis and the thermal and chemical properties are presented in Section 3. The implications of gas cooling and metal redistributions are discussed in Section 4, and our main conclusions are summarized in Section 5. Uncertainties reported are quoted at the $68 \%$ confidence level throughout this work.

\section{Observations and Data Reductions}

\subsection{XMM-Newton}

We include all the existing XMM-Newton observations within $1^{\circ}$ of NGC 1399 as listed in Table 1. Basic data reductions including screening and background modeling were performed using the Science Analysis System (SAS) version xmmsas-20160201. All the ODF files were processed using emchain and epchain to ensure the latest calibrations. Soft flares were filtered from MOS data and pn data using the XMM-ESAS tools mos-filter and pn-filter respectively (Snowden \& Kuntz 2013). The effective exposure time of each detector is listed in Table 1. The combined exposure time is $\approx 100 \mathrm{ks}$ at the center and $\gtrsim 200 \mathrm{ks}$ at the outskirts. We only include events files with FLAG $=0$ and PATTERN $<=12$ for MOS data and with FLAG $=0$ and PATTERN $<=4$ for pn data. Point sources detected by edetect_chain and confirmed by eye were excluded from further analysis. Out-of-time pn events were removed from both spectral and imaging analyses. Modeling of the astrophysical background (AXB) and the Non-X-ray background (NXB) is presented in the Appendix.

Spectral fit of regions of interest was restricted to the $0.5-7.0 \mathrm{keV}$ energy band. These spectra were fit to two sets of models simultaneously. The first model set takes the form of phabs $\times\left(\right.$ pow $_{C X B}+$ ape $_{M w}+$ vape $\left._{\text {ICM }}\right)+$ ape $C_{L B}$. Parameters of the AXB models were fixed to the values listed in Table 3, which were determined with offset pointings. The thermal vapecicm model is for the cluster emission. The abundances of $\mathrm{O}, \mathrm{Ne}, \mathrm{Mg}, \mathrm{Si}, \mathrm{S}, \mathrm{Fe}$, and $\mathrm{Ni}$ were allowed to vary freely; all other elements were tied to Fe. The second model set is to characterize the NXB components (Table 3 ) and their spectra were not folded through the Auxiliary Response Files (ARF). Parameters of the NXB models were fixed to the best fits determined for each observation.

We created images in the $0.5-2.0 \mathrm{keV}$ energy band. Individual detector images were created using the tasks mos-spectra and pn-spectra. Point sources detected by the cheese routine were removed. We used the XMMESAS tasks comb and adapt_900 to create a backgroundsubtracted, vignetting-corrected EPIC mosaic image, binned by a factor of 2 and adaptively smoothed with a minimum of 50 counts per bin. The resulting image is shown in Figure 1 (left).

\subsection{Chandra}

We analyzed a combination of $250 \mathrm{ks}$ Chandra observations centered on NGC 1399 (Obs-ID: 319, 4172, 9530, 14527, 14529, and 16639). We refer interested readers to Paper 1 for details of the observations, data preparation, and the deprojected spectral analysis. All the data were reduced using CIAO 4.9 and $C A L D B$ 4.6.9 following standard procedures. We produced the blank-sky background-subtracted, exposurecorrected, and point source removed image, which covers the entire weak cool core $\left(r<25 \mathrm{kpc} ; t_{\text {cool }}<7.7 \mathrm{Gyr}\right)$, as presented in Figure 2 (top left). In order to enhance detailed structures in the X-ray surface brightness, we divide this $0.5-2.0 \mathrm{keV}$ image by its best-fit double- $\beta$ model. The resulting residual image is shown in Figure 2 (top right). Readout artifacts were subtracted in both imaging and spectral analyses. Spectral fits were performed with XSPEC 12.7 and C-statistics; the solar abundance standard of Asplund et al. (2006) was adopted. We use the thermal emission model vapec to model the hot 
Table 1

XMM-Newton Observational Log of the Fornax Cluster

\begin{tabular}{|c|c|c|c|c|c|c|c|}
\hline Obs ID & Name & Obs-Date & Exposure $(\mathrm{ks})^{\mathrm{a}}$ & R.A. (J2000) & Decl. (J2000) & Offset $\left({ }^{\prime}\right)$ & PI \\
\hline 0012830101 & NGC 1399 & 2001 Jun 27 & $29(4,4,3)$ & 033829.30 & -352701.0 & 0.03 & Buote \\
\hline 0055140101 & LP 944-20 & 2001 Jan 07 & $51(42,43,40)$ & 033934.60 & -352551.0 & 13.41 & Martin \\
\hline 0140950201 & NGC 1386 & 2002 Dec 29 & $17(16,16,14)$ & 033645.40 & -355957.0 & 39.04 & Guainazzi \\
\hline 0210480101 & RX J0337-3457 & 2005 Jan 04 & $49(43,44,41)$ & 033724.70 & -345729.0 & 32.33 & Stanford \\
\hline 0304940101 & NGC 1404 & $2005 \mathrm{Jul} 30$ & $55(22,13,17)$ & 033851.92 & -353539.8 & 9.81 & Matsushita \\
\hline 0400620101 & NGC 1399 & 2006 Aug 23 & $130(81,90,51)$ & 033829.10 & -352703.0 & 0.03 & Paerels \\
\hline 0550930101 & Fornax offset A & 2008 Jun 28 & $14(11,11,8)$ & 033902.40 & -350155.2 & 26.02 & Matsushita \\
\hline 0550930201 & Fornax offset B & 2008 Jun 27 & $17(8,8,4)$ & 033926.16 & -344937.2 & 39.20 & Matsushita \\
\hline 0550930301 & Fornax offset $\mathrm{C}$ & 2008 Jul 17 & $17(11,12,9)$ & 034027.12 & -345916.8 & 36.77 & Matsushita \\
\hline 0550930401 & Fornax offset D & 2009 Feb 09 & $19(14,15,13)$ & 034124.96 & -351004.8 & 39.70 & Matsushita \\
\hline 0550930501 & Fornax offset E & 2009 Feb 23 & $19(18,18,17)$ & 034140.80 & -352251.6 & 39.30 & Matsushita \\
\hline 0550930601 & Fornax offset $\mathrm{F}$ & 2009 Feb 24 & $21(18,18,15)$ & 034135.04 & -353748.0 & 39.35 & Matsushita \\
\hline 0550930701 & Fornax offset $\mathrm{G}$ & 2009 Feb 24 & $19(2,4,1)$ & 034052.08 & -355002.4 & 37.07 & Matsushita \\
\hline 0550931001 & Fornax offset $\mathrm{J}$ & 2008 Jun 25 & $22(18,19,14)$ & 034140.80 & -352251.6 & 39.30 & Matsushita \\
\hline 0550931201 & Fornax offset L & 2008 Jun 25 & $13(8,8,4)$ & 033615.60 & -353256.4 & 27.79 & Matsushita \\
\hline 0550931401 & Fornax offset $\mathrm{N}$ & 2008 Jun 26 & $12(11,11,9)$ & 033711.52 & -351734.8 & 18.41 & Matsushita \\
\hline 0694670101 & NGC 1380 ULX & 2013 Jan 25 & $103(69,70,59)$ & 033625.20 & -345918.0 & 37.53 & Sarazin \\
\hline
\end{tabular}

Note.

${ }^{\text {a }}$ Effective exposure times of MOS1, MOS2, and pn are listed in the brackets.

gas component and a power-law model with an index of 1.6, $\mathrm{pow}_{1.6}$, to describe the unresolved low-mass X-ray binaries (LMXB; Irwin et al. 2003). The deprojected temperature is below $1 \mathrm{keV}$ at the cluster center and rises to $1.5 \mathrm{keV}$ beyond $10 \mathrm{kpc}$.

Within its strong cool core $\left(r<4.5 \mathrm{kpc} ; t_{\text {cool }}<1 \mathrm{Gyr}\right)$, the contribution to the X-ray emissions from the diffuse stellar emission and unresolved LMXB may become progressively more important. We calibrate their contribution to the X-ray surface brightness based on the $K$-band surface brightness profile using the Two Micron All Sky Survey (2MASS; Skrutskie et al. 2006) archived image (see Su et al. 2017a), which amounts to about $1 \%$ of the total X-ray luminosity. We then subtracted the diffuse stellar emission and unresolved LMXB emission from the $0.5-2.0 \mathrm{keV}$ image as shown in Figure 3 (top left).

\section{Analysis and Results}

\subsection{Spectroscopic Maps}

With Chandra observations, we performed a two-dimensional spectroscopic analysis using the Weighted Voronoi Tesselation (WVT) binning (Diehl \& Statler 2006) based on the Voroni binning algorithm presented in Cappellari \& Copin (2003). We generated a WVT binning image containing 137 regions for the Chandra image in the $0.5-2.0 \mathrm{keV}$ band (Figure 2 top left). Each bin has an $\mathrm{S} / \mathrm{N}$ of 80 . We applied a model containing two-temperature components to probe its gas metallicity distribution, otherwise "Fe-bias" would be caused by fitting a single thermal model to multi-phase gas (Buote 2000). The two-temperature model takes the form of phabs $\times\left(\right.$ vapec + vapec pow $\left._{1.6}\right)$. The metallicities of the two vapec components were linked to each other. The twotemperature fit cannot be well constrained for all regions and we find it necessary to fix one temperature at $1.5 \mathrm{keV}$. The other temperature and the normalizations are allowed to vary independently. The resulting $\mathrm{Fe}$ abundance map is shown in Figure 2 (bottom left). The southwest side of NGC 1399 is more metal-enriched than the northeast side and the metal distribution traces the spiral morphology of the sloshing front.

To probe the thermal structure of the hot gas within its strong cool core $(r<4.5 \mathrm{kpc})$, we produced a binned image containing 126 regions for the image in the $0.5-2.0 \mathrm{keV}$ band (Figure 3, top left). Each bin has an $\mathrm{S} / \mathrm{N}$ of 36 . The spectra were fit with the model phabs $\times\left(\right.$ apec pow $\left._{1.6}\right)$. The abundance was fixed to the solar abundance, which is approximately the average metal abundance at the cluster center. The resulting temperature map is presented in Figure 3 (bottom left).

\subsection{Gas Sloshing}

Sloshing cold fronts are identified by eye in images and confirmed by abrupt changes in surface brightness and temperature. The XMM-Newton mosaic image as shown in Figure 1 (left) reveals several edges in the X-ray surface brightness in Fornax: the outermost one is at $200 \mathrm{kpc}\left(30^{\prime}\right)$ to the east $\left(\mathrm{F} 1\right.$, red), the second outermost one at $30 \mathrm{kpc}\left(5^{\prime}\right)$ to the southwest (F2, magenta), and an inner one at about $10 \mathrm{kpc}\left(2^{\prime}\right)$ to the northeast (F3, blue). These fronts stand out in the matching residual image as shown in Figure 1 (right), obtained by dividing the XMM-Newton image with its azimuthal average. Together, they form a spiral pattern wrapping around the bright cluster core. The deep Chandra observation covers F3 and the extended emission to the southwest (Figure 2). Chandra reveals another possible front at $\lesssim 3 \mathrm{kpc}(0,5)$ to the west (F4, black) as shown in Figure 3 (top left). We present the surface brightness profiles over a radial range of $1-250 \mathrm{kpc}$ in the northeast and southwest directions (Figure 4). All four edges can be identified spanning two orders of magnitude in radius. These features are suggestive of gas sloshing triggered by perturbations of minor mergers or off-axis mergers on a large scale.

We present the temperature profiles of the east and southwest directions crossing the outer fronts in Figure 5. The two edges, $\mathrm{F} 1$ and F2, are associated with cooler gas relative to the gas at the same radii but on the opposite side of the cluster. The northeast front at $r=10.6 \mathrm{kpc}\left(116^{\prime \prime}\right), \mathrm{F} 3$, is the most evident 

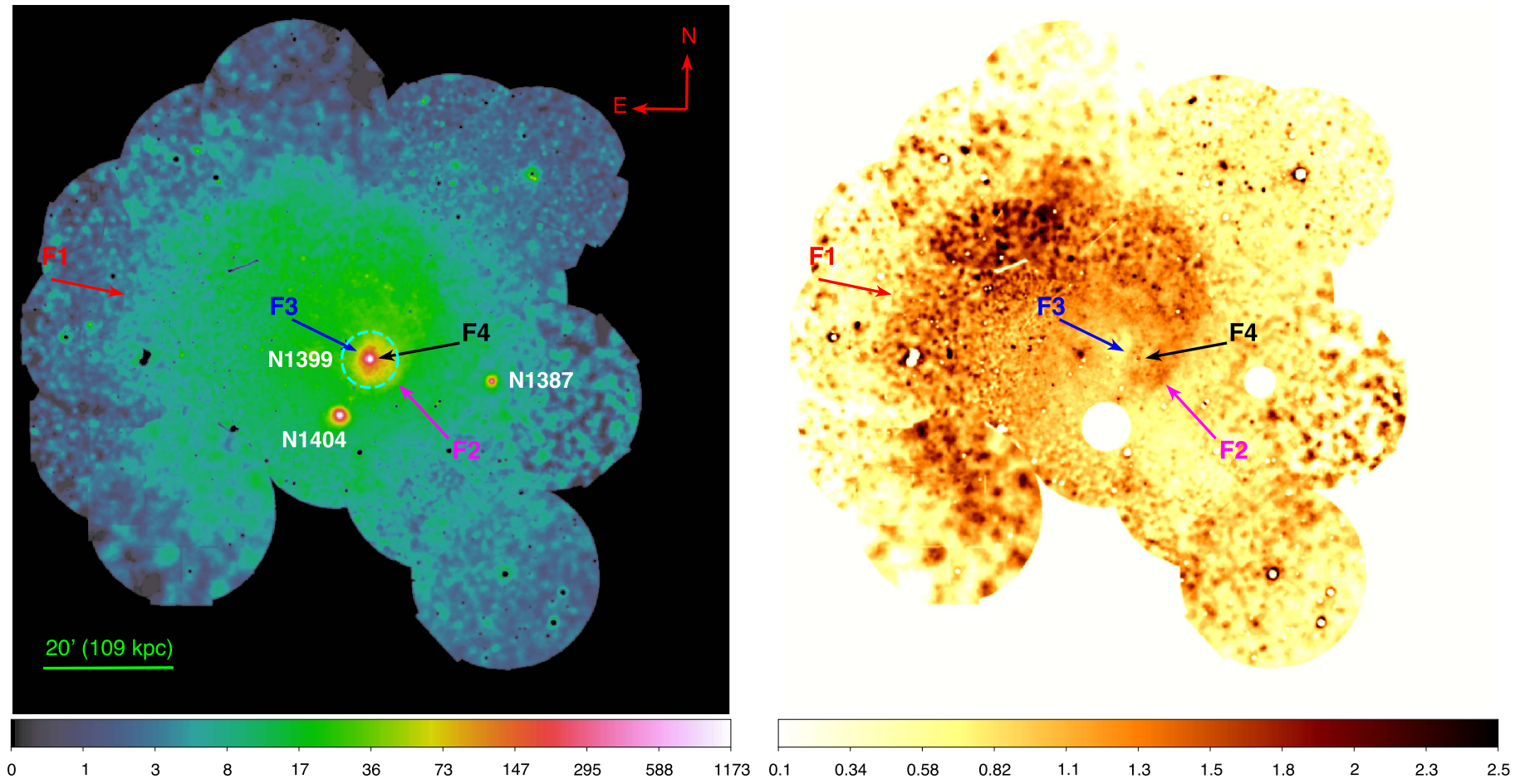

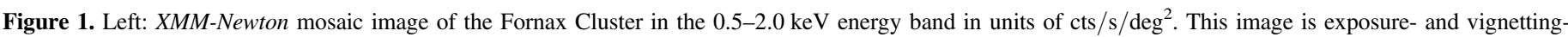

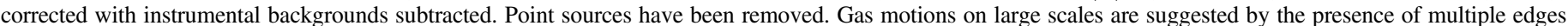

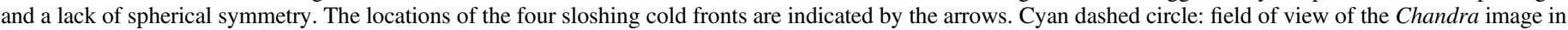
Figure 2. Right: The matching residual image of the left image with respect to the azimuthal average.

front (Figure 2, top left). We apply a Gaussian Gradient Magnitude (GGM) filter to highlight sharp edges in the Chandra X-ray image, which determines the magnitude of surface brightness gradients using Gaussian derivatives (Sanders et al. 2016a; Walker et al. 2016). Brighter regions correspond to sharp features in surface brightness. The resulting GGM image is shown in Figure 2 (bottom right), obtained by combining images on scales of $2 \sigma, 4 \sigma, 8 \sigma, 16 \sigma$, and $32 \sigma$. F3 stands out as a sharp edge at $r=10 \mathrm{kpc}$ to the northeast on the GGM image. We compare the Chandra X-ray surface brightnesses derived in annular sectors with a radial bin width of $2^{\prime \prime}$ from the cluster center to the northeast $\left(133^{\circ}-159^{\circ}\right.$, marked in the red sector in Figure 2, top right) and to the southwest $\left(290^{\circ}-390^{\circ}\right.$, marked in the black sector in Figure 2, top right). As shown in Figure 6, the surface brightness profile of the southwest sector declines more smoothly than that of the northeast sector. We fit a broken power-law density model to the northeast profile and we obtain a break at $116 \pm 1^{\prime \prime}$ $(10.6 \mathrm{kpc})$ relative to the cluster center, which corresponds to a gas density jump of $2.1 \pm 0.2$. We convolve this power-law density model with a Gaussian component and obtain a best-fit width of $\sigma=5^{\prime \prime}(450 \mathrm{pc})$. The smoothed model does not provide a better fit with a $F$-test probability of 0.11 . We extracted spectra from seven concentric annular sectors across this northeast edge (marked in the red annular sectors in Figure 2, top right). The spectra were fit to a single-temperature model. For comparison, we performed the same analysis for the southwest direction (marked in the black annular sectors in Figure 2, top right). The resulting temperature profiles are presented in Figure 6. The gas on the brighter side of the northeast edge is cooler than that on the fainter side, suggesting that this edge is a cold front. The Fe abundance is higher within the edge than that outside as shown in Figure 2 (bottom left).
Hot, diffuse, and relatively pristine cluster gas from the larger radii may have been brought into contact with the cool, dense, and enriched gas by sloshing. The distribution of the gas to the southwest over the same radial range displays a relatively uniform thermal and chemical distribution.

The X-ray emitting structure within the strong cool core of Fornax, tracing the pure hot gas distribution, is shown in Figure 3 (top left). We note a surface brightness discontinuity to the west with a best-fit edge at $r=2.9 \mathrm{kpc}$ (Figure 7-top), while the surface brightness profile to the east is relatively smooth. We derive the temperature profile across this west edge as shown in Figure 7 (bottom). It rises abruptly from $\sim 1 \mathrm{keV}$ to $\sim 1.5 \mathrm{keV}$ over a radial range of $1-2 \mathrm{kpc}$, manifesting the presence of a cold front, consistent with the temperature map (Figure 3-bottom-left). In contrast, the temperature profile over the same radial range to the east varies from $\sim 1.1 \mathrm{keV}$ to $\sim 1.2 \mathrm{keV}$.

\subsection{Subkiloparsec Structures}

We produce a GGM image to probe substructures in the innermost $5 \mathrm{kpc}$ by combining GGM images on scales of $1 \sigma$, $2 \sigma, 4 \sigma, 8 \sigma$, and $16 \sigma$. As shown in Figure 3 (top right), a subkiloparsec region of enhanced surface brightness is visible just outside the cold front (F4) to the west (blue box). Two 3-5 kpc long bright filaments can be identified to the east at a similar radius (Figure 3 green and magenta boxes). We perform spectral analysis for these structures to determine their natures. We apply local background spectra extracted from neighboring regions as shown in Figure 3 (top left; cyan dashed box for S1; cyan dashed circle for $\mathrm{S} 2$ and $\mathrm{S} 3$ ). The spectra were fit to the model phabs $\times\left(\right.$ apec $\left.+\mathrm{pow}_{1.6}\right)$. Their metal abundance was fixed at the solar abundance. The best-fit temperatures of S2 

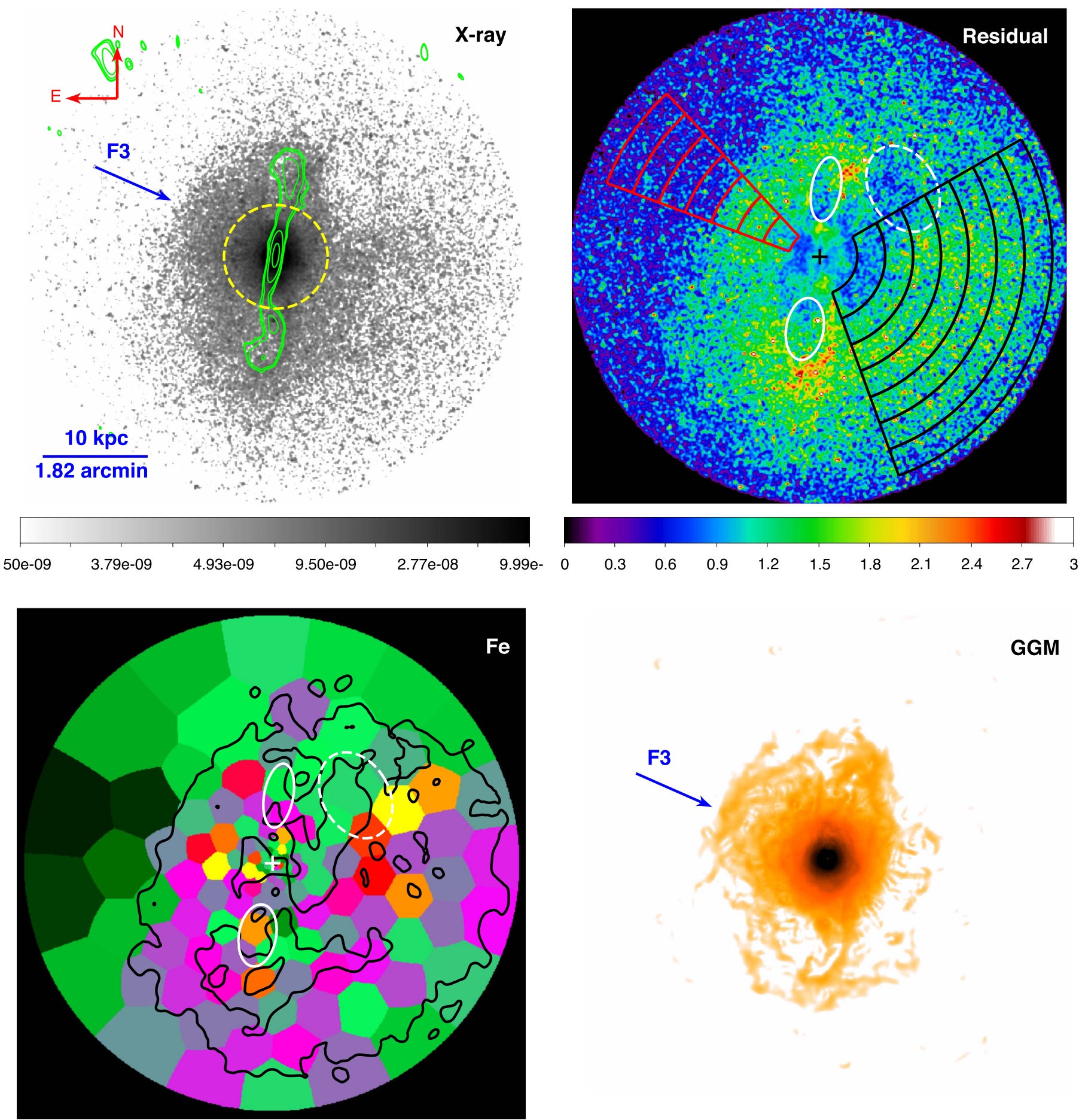

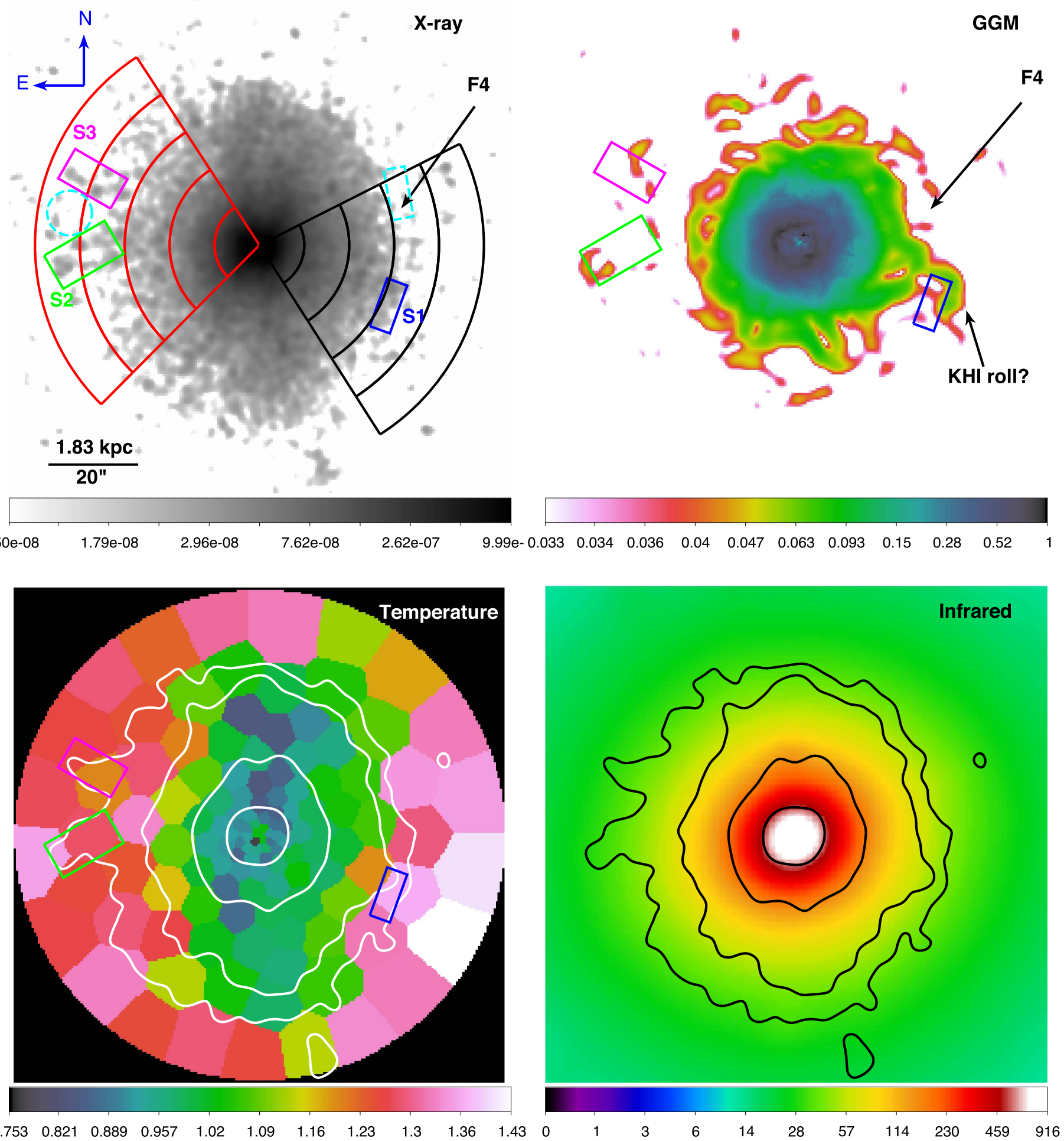

$\begin{array}{llllllllllll}0.753 & 0.821 & 0.889 & 0.957 & 1.02 & 1.09 & 1.16 & 1.23 & 1.3 & 1.36 & 1.43 & 0\end{array}$

Figure 3. Gas properties within the strong cool core of Fornax $\left(r<4.5 \mathrm{kpc} ; t_{\text {cool }}<1 \mathrm{Gyr}\right)$, corresponding to the yellow dashed circle in Figure 2 (top left). Top left: Chandra X-ray image within a radius of 50" $(4.5 \mathrm{kpc})$ with stellar and LMXB components subtracted. Positions of three structures are marked in blue, green, and magenta boxes. Spectra extracted from cyan dashed regions are used as the local background for the spectral fit of S1, S2, and S3. Top right: Gaussian Gradient Magnitude filtered image of the top-left image, obtained by combining images on scales of $1 \sigma, 2 \sigma, 4 \sigma, 8 \sigma$, and $16 \sigma$. Bottom left: two-dimensional temperature distribution of the hot gas in the innermost region of NGC 1399 in units of kiloelectron volts. White contours: Chandra X-ray emission in the 0.5-2.0 keV energy band. Bottom right: WISE infrared image with X-ray contours overlaid. Note that there is no offset between the X-ray and the infrared centroids, implying that Fornax did not experience any recent disturbance.

pressure of the ambient ICM using the deprojected density profile of NGC 1399 determined in Paper 1. S3 is in pressure equilibrium with the ambient ICM. S2 would be overpressurized unless its temperature is near its lower limit of $\sim 1 \mathrm{keV}$. The temperature of S1 cannot be constrained. Assuming $\mathrm{S} 1$ is also in pressure equilibrium, its temperature would be $\sim 1 \mathrm{keV}$. The low temperature (cooler than the ambient ICM) of these subkiloparsec features meets our 


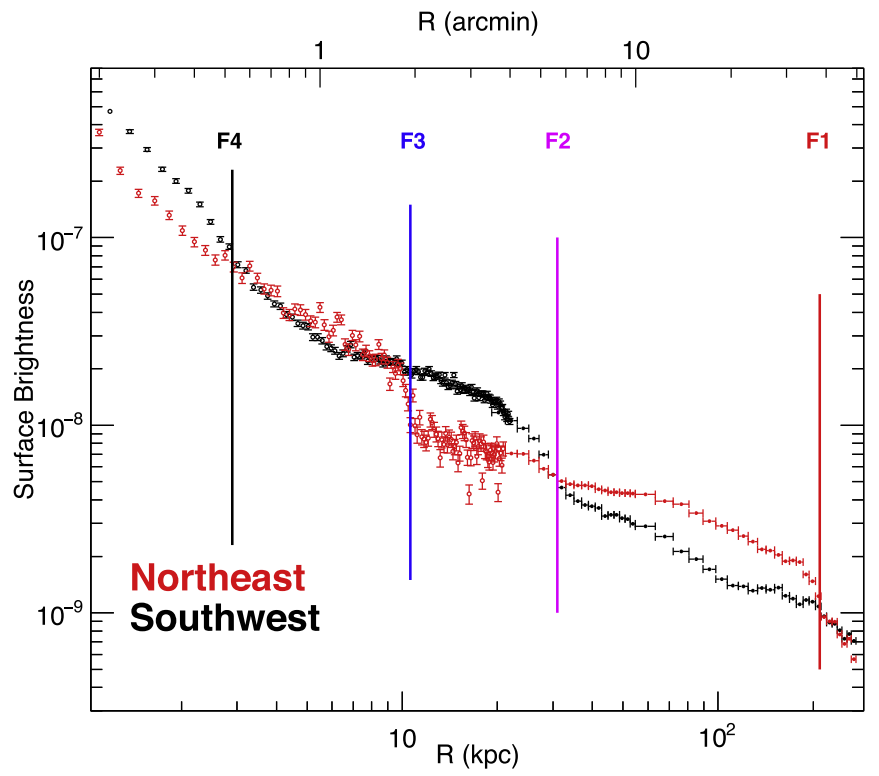

Figure 4. Alternating pattern of the surface brightness profiles in the $0.5-2.0 \mathrm{keV}$ energy band of the northeast (red) and the southwest (black) directions over a radial range of $1-250 \mathrm{kpc}$. The data points were derived with Chandra (small radii, open circles) and XMM-Newton (large radii, filled circles) observations and in units of photon $\mathrm{cm}^{-2} \mathrm{~s}^{-1} \operatorname{arcsec}{ }^{-2}$. Vertical solid lines mark the positions of surface brightness edges.

expectation for KHI eddies growing at cold fronts (e.g., NGC 1404, Su et al. 2017a). Their properties and locations are also consistent with being low-entropy filaments induced by thermal instability (e.g., NGC 5044, David et al. 2014, 2017).

\section{Discussion}

We perform a joint Chandra and XMM-Newton analysis of the Fornax Cluster. A series of sloshing cold fronts (F1, F2, F3, and F4) are present in the ICM. Nearly all of them fall on the same spiral structure, spanning from a radius of $200 \mathrm{kpc}$ to the cooling radius. We discuss their constraints on the cluster merging history and the effect of gas sloshing in the chemical and thermal distribution of the ICM.

\subsection{Merging History Recorded by Sloshing Cold Fronts}

We identify four edges in the X-ray surface brightness at radii of $200 \mathrm{kpc}, 30 \mathrm{kpc}$ (Figures 1 and 5), $10 \mathrm{kpc}$ (Figures 2 and 6), and $3 \mathrm{kpc}$ (Figures 3 and 7), all distributed along the SW-NE direction (Figures 1 and 4). The brighter sides of these edges comprise cooler gas. We infer that they are sloshing cold fronts induced by minor or offset mergers. Gas motion around the cluster center approximates the oscillating flow in a statically stable environment. We calculate the Brunt-Väsälä frequency (buoyancy frequency; Cox 1980) at each radius, $r$ (Balbus \& Soker 1990):

$$
\omega_{\mathrm{BV}}=\Omega_{\mathrm{K}} \sqrt{\frac{1}{\gamma} \frac{d \ln K}{d \ln r}},
$$

where $K=k T / n^{2 / 3}$ is the gas entropy, $\Omega_{\mathrm{K}}=\sqrt{\frac{G M}{r^{3}}}$ is the Keplerian frequency, and $\gamma=5 / 3$. The density and temperature profiles of the cluster center, measured with Chandra observations, are used to calculate $K$ and to derive the

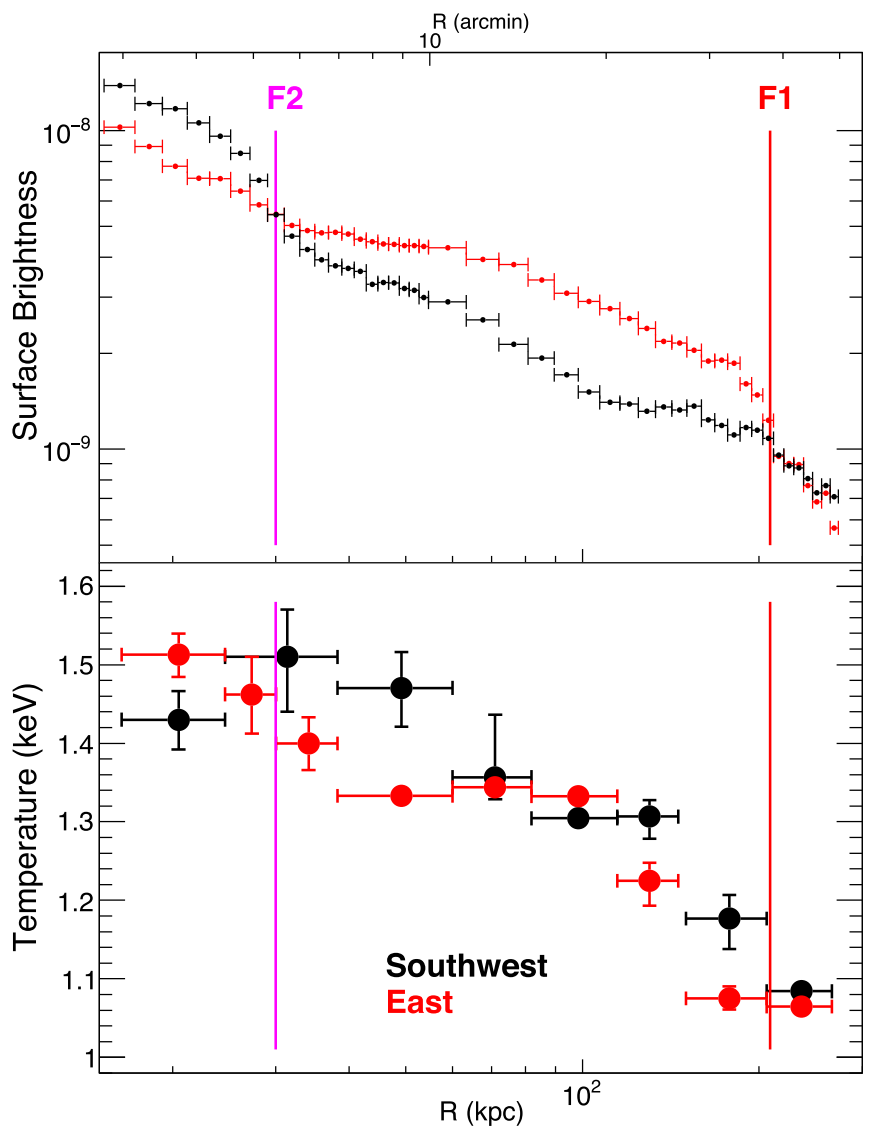

Figure 5. XMM-Newton surface brightness (in the $0.5-2.0 \mathrm{keV}$ energy band and in units of photon $\mathrm{cm}^{-2} \mathrm{~s}^{-1}$ arcsec ${ }^{-2}$; top panel) and projected temperature (bottom panel) profiles of the northeast (red) and the southwest (black) directions outside the cluster center, over a radial range of $3^{\prime}-41^{\prime}(15-250 \mathrm{kpc})$. Vertical lines mark the location of the cold fronts F1 and F2.

hydrostatic mass profile, $M(r)$; for comparison, we also derive the total mass using the stellar velocity dispersion (Paper 1). Gas properties outside the weak cool core are measured with XMM-Newton; the best-fit Navarro-Frenk-White (NFW) dark matter profile of Navarro et al. (1997) is used to calculate $\Omega_{\mathrm{K}}$. The resulting sloshing period of $P=2 \pi / \omega_{\mathrm{BV}}$ is shown in Figure 8 , which increases with radius. The sloshing timescale $(P$ or $P / 2)$ provides an order-of-magnitude estimate of the age of the sloshing front (Churazov et al. 2003). To verify this approximation, we compare these timescales to the evolution of the sloshing simulated for a Virgo-like cluster (Figure 7 in Roediger et al. 2011). The ages of the simulated sloshing fronts at radii of $100 \mathrm{kpc}(\mathrm{NW}), 40 \mathrm{kpc}(\mathrm{SE})$, and $20 \mathrm{kpc}(\mathrm{NW})$ are $1.7 \mathrm{Gyr}$, $0.8 \mathrm{Gyr}$, and $0.6 \mathrm{Gyr}$, respectively (fronts within $10 \mathrm{kpc}$ are not resolved). These scales are comparable to the sloshing fronts observed in Fornax in this work at $200 \mathrm{kpc}(\mathrm{E})$, $30 \mathrm{kpc}(\mathrm{SW})$, and $10 \mathrm{kpc}(\mathrm{NE})$ with their ages $(P / 2 \sim P)$ corresponding to $2.5-5.0 \mathrm{Gyr}, \quad 0.4-0.7 \mathrm{Gyr}, \quad 0.1-0.2 \mathrm{Gyr}$, respectively, as shown in Figure 8. Our approach thus leads to reasonable approximations. In future work, we plan to refine our estimates with simulations specifically tailored to Fornax (A. Sheardown et al. 2017, in preparation).

Simulations reveal that the infall of one subcluster is capable of inducing multiple sloshing cold fronts as the displaced gas peak oscillates back and forth around the dark matter center of 


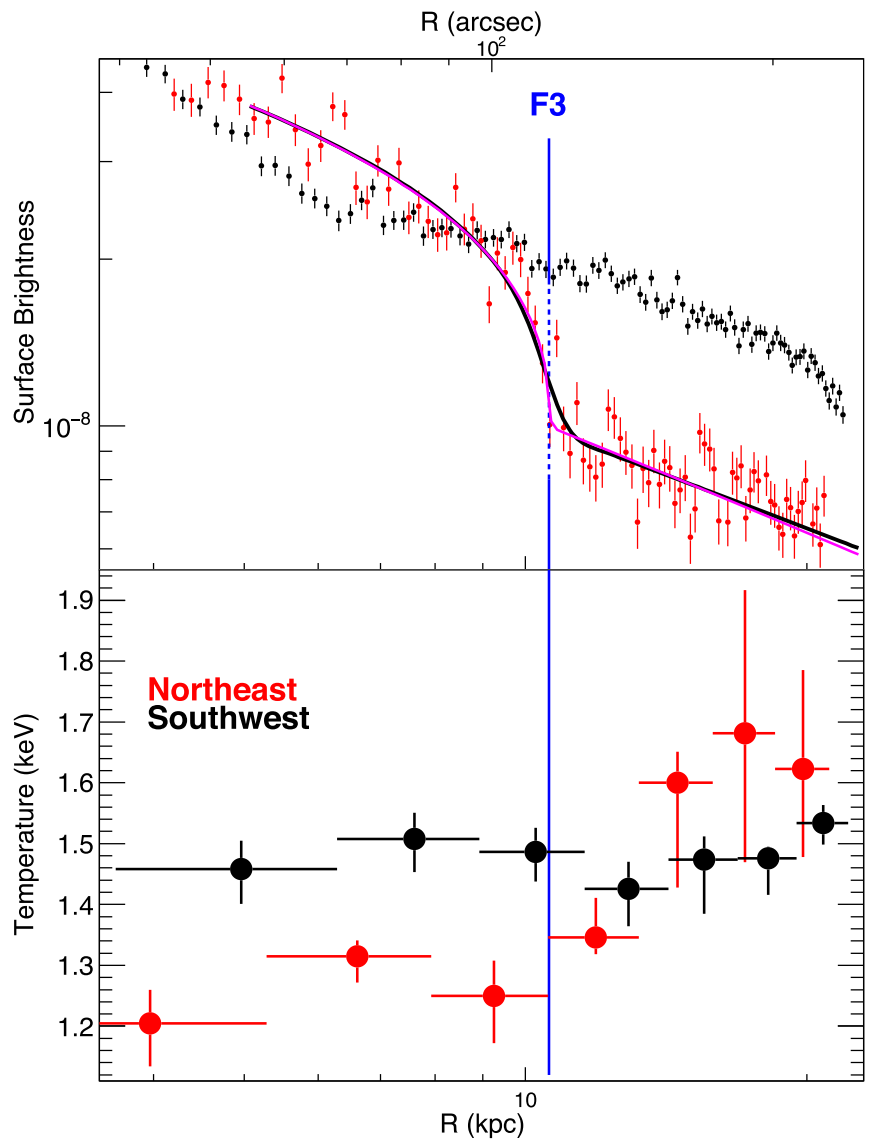

Figure 6. Top: Chandra surface brightness profiles in the $0.5-2.0 \mathrm{keV}$ energy band in the northeast (red) and southwest (black) directions relative to the cluster center and in units of photon $\mathrm{cm}^{-2} \mathrm{~s}^{-1} \operatorname{arcsec}^{-2}$. A sharp edge, marked by the blue line, can be identified in the northeast direction at $R=116^{\prime \prime}$ indicating the presence of a sloshing cold front, while the hot gas is smoother and more extended in the southwest direction. Magenta line: the best-fit broken power-law density model corresponding to a density jump of 2.1. Black line: best-fit broken power-law density model smeared with the Gaussian $\sigma=5^{\prime \prime}$. Bottom: projected temperature profiles in the northeast and southwest directions, respectively (red and black sectors in Figure 2, top right) measured with Chandra. Radius is relative to the cluster center.

the main cluster (ZuHone et al. 2010, 2011). That F1, F2, and F3 fall on the same spiral structure is typical for sloshing cold fronts produced in a single merger event, as seen in simulations (e.g., Roediger et al. 2011). NGC 1404 and NGC 1387 are the second and third brightest member galaxies in Fornax (Figure 1). NGC 1387 is more than $5 \times$ fainter in X-rays than NGC 1404 and its radial velocity relative to NGC 1399 is $\gtrsim 100 \mathrm{~km} \mathrm{~s}^{-1}, 5 \times$ smaller than that of NGC 1404. Therefore, the infall of NGC 1404 may have initiated the gas sloshing. Adopting a timescale of $P / 2 \sim P=2.5 \sim 5 \mathrm{Gyr}$ for the outermost front at $r=200 \mathrm{kpc}$ (Figure 8), we estimate that the infall of NGC 1404, took place more than 2 Gyr ago, longer than the typical crossing time of galaxy clusters. This is consistent with our previous study of NGC 1404 approaching the inner region of Fornax for the second time (Su et al. 2017c). Fornax is one of the few clusters where the perturber that has initiated the sloshing can be identified, providing an ideal clean case for tailored simulations.

\subsection{Metallicity Redistribution at the Cluster Center}

The two-dimensional $\mathrm{Fe}$ abundance distribution of NGC 1399 (Figure 2, bottom left) shows that the hot gas with

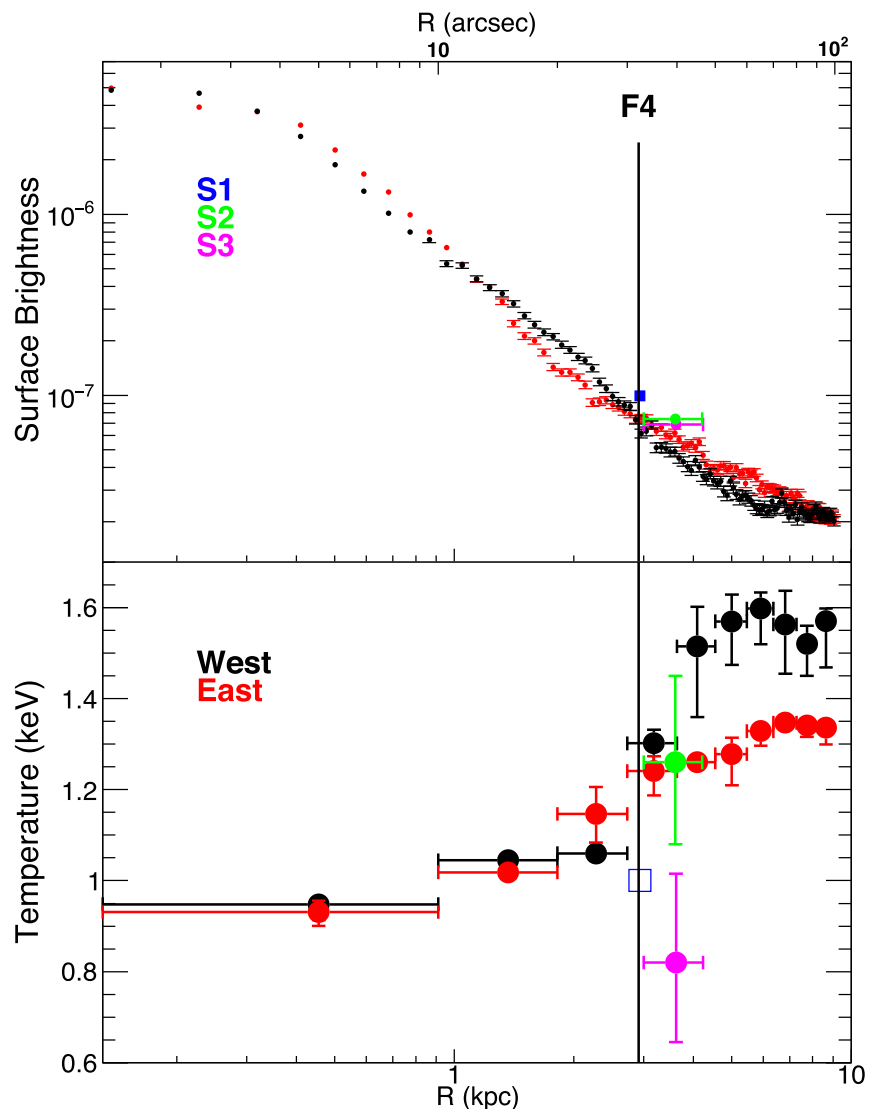

Figure 7. Chandra surface brightness (in the $0.5-2.0 \mathrm{keV}$ energy band and in units of photon $\mathrm{cm}^{-2} \mathrm{~s}^{-1}$ arcsec ${ }^{-2}$ ) (top panel) and projected temperature (bottom panel) profiles of the east (red) and the west (black) directions within a radius of $100^{\prime \prime}(9.1 \mathrm{kpc})$. The corresponding regions of the inner five data points on the temperature profile are marked in Figure 3 (top left). The location of a possible sloshing cold front is marked by the black line. The three structures (Figure 3) are marked in blue (S1), green (S2), and magenta (S3) symbols; their temperatures are derived with local background. The temperature of S1 is not constrained in the spectral fit. We derive its temperature (blue open square) by assuming $\mathrm{S} 1$ is in pressure equilibrium with the ambient ICM.

enhanced metallicity $\left(>1.0 Z_{\odot}\right)$ reaches radii of approximately $5-10 \mathrm{kpc}$ to the north and the east and it is more extended to the south and the west, reaching beyond $12 \mathrm{kpc}$. The extended distribution of enriched ICM may reflect an AGN outburst or gas sloshing. The enriched gas to the north is most likely due to the AGN outburst along the north-south direction. Kirkpatrick \& McNamara (2015) calibrated an empirical relation between the maximum projected radius of the uplifted gas and the cavity enthalpy

$$
R_{\mathrm{Fe}}=(57 \pm 30) \times H^{0.33 \pm 0.08} \mathrm{kpc},
$$

where $H$ is in units of $10^{59} \mathrm{erg}$. Substituting the cavity enthalpy of NGC 1399 (Paper 1), we obtain a $R_{\mathrm{Fe}}$ of $3-10 \mathrm{kpc}$, consistent with the observed enriched gas extent to the north. The enriched gas extended from the south to the west follows the spiral pattern of the gas sloshing, strongly suggesting that gas sloshing is driving the metal redistribution at the center cluster.

While AGN bubbles are very vulnerable to gas motions at the cluster center (Morsony et al. 2010), the two bubbles in NGC 1399 are remarkably symmetric and intact in both X-ray and radio. We thus infer that the AGN outburst has taken place 


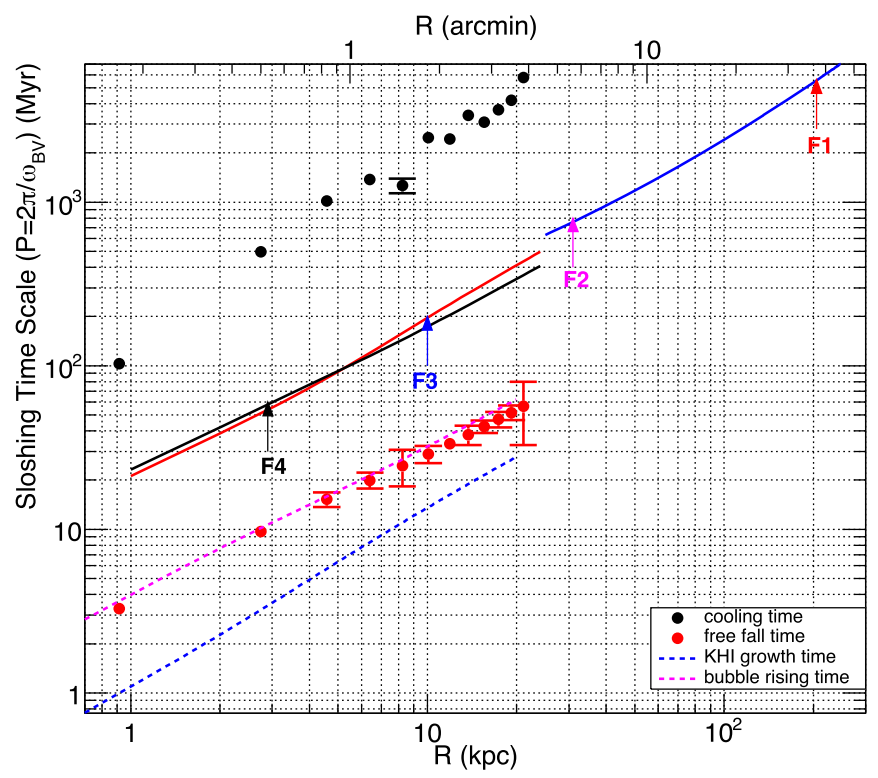

Figure 8. Sloshing timescale (oscillation period, $P=2 \pi / \omega_{\mathrm{BV}}$, where $\omega_{\mathrm{bv}}$ is the Brunt-Väsälä frequency) as a function of radius, compared to the cooling time (black circles) and the free-fall time (red circles). Data points within $r=20 \mathrm{kpc}$ are obtained with Chandra and those outside $r=30 \mathrm{kpc}$ are with XMMNewton. Dark black, red, and blue solid lines are derived using hydrostatic mass, velocity dispersion mass, and the best-fit NFW mass, respectively. Arrows mark the positions of the four sloshing fronts found in Fornax.

later than the gas sloshing. This is consistent with the cycle of AGN outbursts (a few tens of megayears) being much shorter than the timescale of gas sloshing $(\sim 1 \mathrm{Gyr}$; ZuHone et al. 2010). The enriched gas along the southwest front is more metal abundant, more extended, and distributed to a larger radius than the enriched gas to the north. We therefore conclude that gas sloshing, rather than AGN uplift, has played a primary role in redistributing the enriched gas, at least in this particular case.

\subsection{Microphysics and Regulation of Cooling}

While the cooling time drops below $1 \mathrm{Gyr}$ at the center of Fornax, as in many more massive cool-core clusters, the star formation rate is negligible in NGC 1399 (Vaddi et al. 2016). AGN feedback is by far the most viable solution to the cooling problem. The mechanical energy provided by $\mathrm{AGN}$ is correlated with the cool-core luminosity (Bîrzan et al. 2004). AGN activity can respond to cooling through precipitation of cooled gas, as proposed by McCourt et al. (2012). Still, it is unclear how the jet power is transformed into thermal energy. One route is by the dissipation in the ICM of turbulence generated in the wakes of rising bubbles (Churazov et al. 2002). Then again, Zhuravleva et al. (2017) found that in the cool cores of some clusters (e.g., the Perseus Cluster and Abell 1795), gas perturbations are associated with gas motion (isobaric) rather than AGN bubbles (isothermal), suggesting that the bulk of the turbulence may not be generated by radio outbursts. Apart from AGN outbursts, gas sloshing has been considered as a means of quenching cooling flows (Fujita et al. 2004; ZuHone et al. 2010). Frequent gas sloshings do not necessarily require a high merging rate in that multiple sloshing fronts can be induced by just one subcluster infall (ZuHone et al. 2010, 2011). NGC 1399 displays a series of sloshing cold fronts at various radii. As demonstrated in Figure 8, the sloshing timescale is $\approx 10 \times$ shorter than the cooling time over the entire cluster center, making it a promising mechanism to heat the cool core. However, it would be far fetched for gas sloshing to be the primary mechanism of preventing the gas from cooling, unless the frequency of gas sloshing responds to the state of the gas. Nevertheless, with AGN feedback being the primary solution, gas sloshing may still provide supplemental heating to the cool core, as long as heat can be transported effectively between gases of different entropy. Below we discuss two transport processes that may occur at the interface.

\subsubsection{Conduction}

Thermal conduction is expected to erase temperature gradients outside the strong cool core $\left(1 \mathrm{Gyr}<t_{\text {cool }}<14 \mathrm{Gyr}\right.$; Voit et al. 2015), where most sloshing cold fronts reside. If conduction is reduced, low-entropy gas brought out by sloshing would eventually fall back to the cluster center (Ghizzardi et al. 2014).

We calculate the characteristic mean-free path (mfp) of electrons, $\lambda_{e}$, in the hot plasma at the leading edge. Sarazin (1988) gives

$$
\lambda_{e}=\frac{3^{3 / 2}\left(k T_{e}\right)^{2}}{4 \pi^{1 / 2} n_{e} q_{e}{ }^{4} \ln \Lambda} .
$$

where $n_{e}$ is the electron density and the Coulomb logarithm is

$$
\ln \Lambda=35.7+\ln \left[\left(\frac{k T_{e}}{1 \mathrm{keV}}\right)\left(\frac{n_{e}}{10^{-3} \mathrm{~cm}^{-3}}\right)^{-1 / 2}\right] .
$$

Equation (3) can be approximated by

$$
\lambda_{e} \approx 340 \mathrm{pc}\left(\frac{k T_{e}}{1 \mathrm{keV}}\right)^{2}\left(\frac{n_{e}}{10^{-3} \mathrm{~cm}^{-3}}\right)^{-1},
$$

assuming the temperatures of electrons, ions, and gas are equal. Substituting a temperature of $1.4 \mathrm{keV}$ and a gas density of $0.004 \mathrm{~cm}^{-3}$ for the northeast cold front at $r=10 \mathrm{kpc}(\mathrm{F} 3$, the most prominent cold front), we obtain a $\lambda_{e}$ of $150 \mathrm{pc}$. This value is smaller than we can resolve in either our imaging or spectral analysis. Therefore, we are unable to strictly determine the effective conductivity in the ICM in this current work. Following Sarazin (1988) and Markevitch et al. (2003), we estimate the timescale for the thermal conduction to operate in the Spitzer regime

$$
\begin{aligned}
t_{\text {cond }} \sim & k l^{2} n_{\mathrm{e}} / \kappa_{\mathrm{s}} \sim 12\left(\frac{n_{e}}{0.002 \mathrm{~cm}^{-3}}\right) \\
& \times\left(\frac{l}{100 \mathrm{kpc}}\right)^{2}\left(\frac{T}{10 \mathrm{keV}}\right)^{-5 / 2} \mathrm{Myr},
\end{aligned}
$$

where $\kappa_{\mathrm{s}}$ is the Spitzer value (1956), $l$ is the size of the cold front, and $T$ is the ICM temperature. Applying the conditions of F3, we obtain $t_{\text {cond }} \lesssim 10 \mathrm{Myr}$, shorter than the age of the bubbles ( $15 \mathrm{Myr}$, Paper 1). As we discussed in Section 4.2, AGN outburst is more recent than the event that caused the sloshing. The northeast sloshing front, residing at $10 \mathrm{kpc}$, has a sloshing timescale of $100 \sim 200 \mathrm{Myr}$ (Figure 8). The conductivity, possibly being reduced by a factor of $\left(\kappa / \kappa_{\mathrm{s}}\right)^{-1} \sim$ $t_{\text {age }} / t_{\text {cond }} \gtrsim 10$, is not sufficient to wipe out temperature gradient. This is consistent with the edge-fitting of the surface brightness profile that convolving the power-law density model 
with a Gaussian component is not required (Section 3.2). Komarov et al. (2014) argue that fluid elements along the presumably incompressible cold front are stretched, which tend to enhance the temperature gradients and align the originally random magnetic field lines along the front. Consequentially, and counterintuitively, heat flux can be reduced at the cold front where the temperature gradient is the largest. Then again, using MHD simulations, ZuHone et al. (2013, 2015) found that the temperature gradient can nevertheless be reduced if the conduction is anisotropic even in the presence of this magnetic layer.

\subsubsection{KHI and Turbulent Mixing}

The innermost sloshing cold front (F4) resides at $r \lesssim 3 \mathrm{kpc}$ to the west while the two AGN bubbles are more than $5 \mathrm{kpc}$ away from the cluster center. Sloshing brings high-entropy gas to the innermost regions where cooling is most severe $\left(t_{\mathrm{cool}}<1 \mathrm{Gyr}\right)$. This innermost sloshing cold front is not sharp with a subkiloparsec bright structure, a KHI eddy candidate, visible at the interface (blue box in Figure 3). Its temperature could be $\approx 1 \mathrm{keV}$, similar to the gas temperature inside the interface.

Either viscosity or ordered magnetic field can damp out the growth of KHI. The presence of a KHI roll allows us to put upper limits on the viscosity and the magnetic field strength using the criteria presented in Roediger et al. (2013) and Vikhlinin et al. (2001), respectively. For a shear flow velocity with a Mach number $\mathcal{M}$, the fraction of the viscosity relative to the Spitzer value, $f_{\nu}$, needs to be smaller than

$$
\begin{aligned}
f_{\nu}< & \frac{10}{16 \sqrt{\Delta}} \cdot \frac{\mathcal{M} c_{s}}{400 \mathrm{~km} \mathrm{~s}^{-1}} \cdot \frac{\ell}{10 \mathrm{kpc}} \\
& \cdot \frac{n_{e, h}}{10^{-3} \mathrm{~cm}^{-3}}\left(\frac{k T_{h}}{2.4 \mathrm{keV}}\right)^{-5 / 2},
\end{aligned}
$$

where $c_{s}\left(=\sqrt{\gamma k T_{h} / \mu m_{p}}\right)$ is the sound speed, $T_{h}$ and $n_{e, h}$ are the temperature and density of the ICM on the high entropy side, $\ell$ is a characteristic length scale, and $\Delta=\frac{\left(\rho_{h}+\rho_{c}\right)^{2}}{\rho_{h} \rho_{c}}$ characterizes the contrast of the gas densities on each side of the interface. This KHI eddy candidate has a height of $h \sim 0.3 \mathrm{kpc}$; we estimate $\ell \approx 2 h \sim 0.6 \mathrm{kpc}$ (Roediger et al. 2013). Likewise, the strength of an ordered magnetic field needs to be smaller than

$$
B<\left[8 \pi \frac{\gamma T_{h} n_{e, h}}{1+T_{c} / T_{h}}\right]^{\frac{1}{2}} \mathcal{M} .
$$

Sloshing cold fronts are found to be subsonic in simulations and the motion slows down toward smaller radii (Ascasibar \& Markevitch 2006; ZuHone et al. 2010, 2011). Roediger et al. (2011) found that the sloshing cold fronts propagate at $\mathcal{M} \sim 0.1$ in a simulation tailored to the Virgo Cluster. We note that there is no offset between the X-ray and the infrared centroids (Figure 3, bottom right), disfavoring a recent sloshing event in Fornax. We assume that this innermost front is $30-60 \mathrm{Myr}$ old, that is the sloshing timescale at $r=3 \mathrm{kpc}$ (Figure 8). This corresponds to an average sloshing velocity of $50-100 \mathrm{~km} \mathrm{~s}^{-1}(\mathcal{M} \lesssim 0.2)$. Here, we conservatively adopt $\mathcal{M}<0.5$ (ZuHone et al. 2011). The upper limits on $f_{\nu}$ and $B$ as a function of $\mathcal{M}$ are presented in Figure 9. A full Spitzer

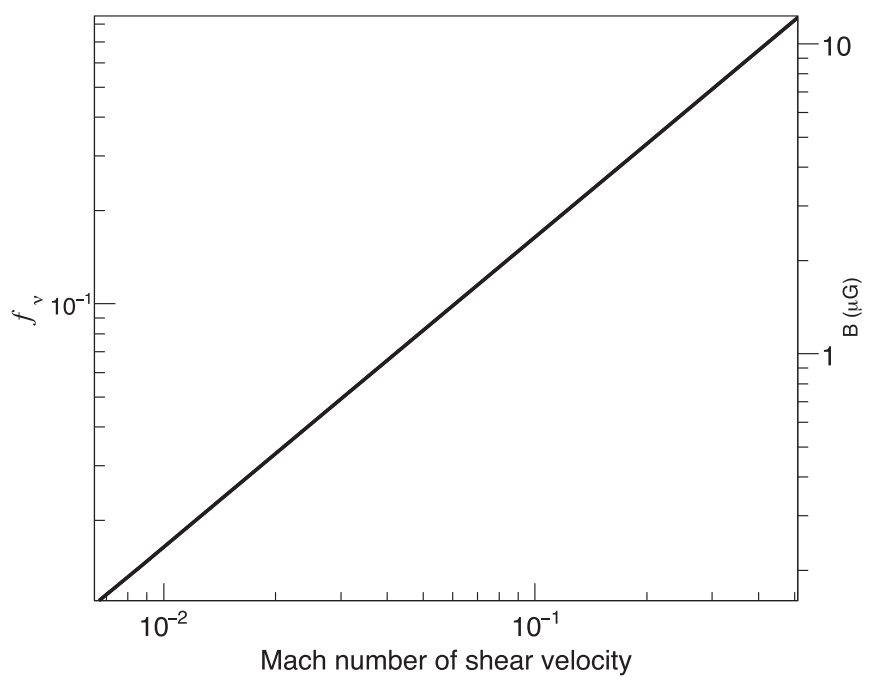

Figure 9. For a given shear velocity along the sloshing front ( $x$-axis), the presence of KHI at the innermost sloshing front in NGC 1399 would be allowed if $f_{\nu}$ (left axis, the fraction of the viscosity relative to the Spitzer value) and $B$ (right axis, the strength of the ordered magnetic field) stay below the black solid line.

viscosity can be ruled out and the strength of an ordered magnetic field should be smaller than $10 \mu \mathrm{G}$. However, as demonstrated in ZuHone et al. (2013), the suppression due to magnetic draping is only partial since the field lines may not be perfectly aligned.

More evident $\lesssim 1 \mathrm{kpc}$ structures are present to the east at a similar radius (green and magenta boxes in Figure 3). In particular, S3 has a best-fit temperature similar to that of the gas within the interface and is in pressure equilibrium with the external gas. We note that the temperature gradient to the east is much smoother than that in the west (Figure 7). This difference may be due to more evident KHI to the east, which may have disrupted a previously existing cold front. KHI accelerates the turbulent mixing of gas of different phases, as we expect from theory and simulations (ZuHone et al. 2011; Roediger et al. 2015a, 2015b). Large KHI rolls (above certain wavelengths, $\ell_{\max }$ ) are expected to be suppressed by gravity (Chandrasekhar 1961). We do not expect to observe KHI rolls larger than

$$
\begin{aligned}
\ell_{\max } \approx & 18 \mathrm{kpc}\left(\frac{\rho_{c} / \rho_{h}}{1.5}\right)^{-1}\left(\frac{U}{200 \mathrm{~km} \mathrm{~s}^{-1}}\right)^{2} \\
& \times\left(\frac{g}{3 \times 10^{-8} \mathrm{~cm} \mathrm{~s}^{-2}}\right)^{-1},
\end{aligned}
$$

where $U$ is the shear velocity at the front and $g$ is the gravitational acceleration (Roediger et al. 2012b). We assume $\rho_{c}=1.5 \rho_{h}$ and $U(r)=0.3 c_{s}(r) ; c_{s}$ and $g(r)$ were calculated from the best-fit deprojected temperature profile and the X-ray hydrostatic mass profile (Paper 1). We present the maximum $\mathrm{KHI}$ length scale as a function of radius at the cluster center in Figure 10. The size of these features stays below this upper limit. A potential ghost bubble of $\gtrsim 5 \mathrm{kpc}$ diameter resides at $r=13 \mathrm{kpc} \quad(r=10 \mathrm{kpc}$ in projection) to the northwest (Figure 2, see Paper 1 for details). Interestingly, Walker et al. (2017) propose that such giant X-ray surface brightness decrement may be due to KHI instead of AGN bubbles. The 


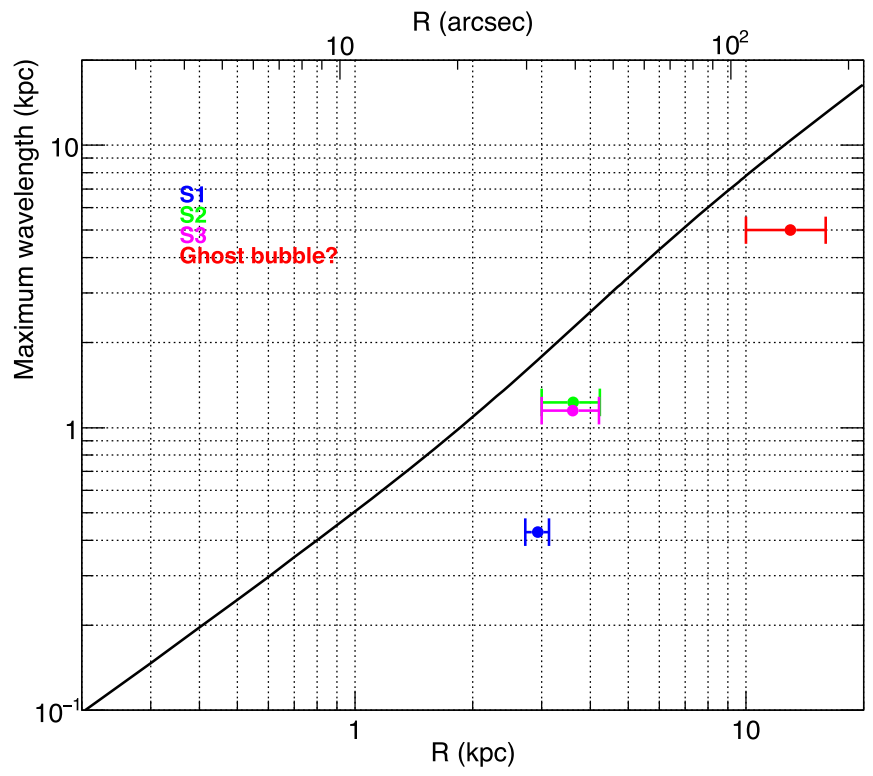

Figure 10. Maximum wavelength for KHI rolls as a function of radius in NGC 1399 (black solid line). Gravity suppresses KHI above this length scale. Blue, green, magenta, and red circles mark the sizes and radii of S1, S2, S3, and a ghost bubble candidate, respectively.

position and size of this ghost bubble candidate are also in the allowed parameter space for a giant KHI roll.

Alternatively, S1, S2, and S3 may be thermally unstable Xray filaments resulting from gas cooling (David et al. 2014, 2017). The growth time of KHI can be estimated from (Roediger et al. 2012a)

$$
\tau_{\mathrm{KH}}=\frac{\sqrt{\Delta}}{2 \pi} \frac{\ell}{U}=3.9 \operatorname{Myr} \sqrt{\Delta} \frac{\ell}{10 \mathrm{kpc}}\left(\frac{U}{400 \mathrm{~km} \mathrm{~s}^{-1}}\right) .
$$

We calculate $\tau_{\mathrm{KH}}$ for the maximum KHI length scale, the maximum growth time, at each radius as shown in Figure 8. The maximum growth time of KHI is shorter than the cooling time by two orders of magnitude. KHI is thus somewhat favored as their origin. The timescale of KHI is also significantly shorter than the dynamical timescale (free-fall time or buoyant rising time), implying that KHI, coupled with gas sloshing, may have played an important role in regulating the thermal state of the hot plasma at cluster centers.

\section{Conclusions}

The Fornax Cluster is a nearby low-mass cool-core cluster. It displays prominent sloshing structure wrapping around the cluster center. We analyzed a mosaic XMM-Newton observation and a total of $250 \mathrm{ks}$ Chandra observations centered on its BCG NGC 1399. We present the properties of the hot gas over a radial range of $1-250 \mathrm{kpc}$. We found the following.

1. Four sloshing fronts are visible at radii of $200 \mathrm{kpc}$, $30 \mathrm{kpc}, 10 \mathrm{kpc}$, and $3 \mathrm{kpc}$ respectively, allowing us to probe the merger history of Fornax. We speculate that all of these fronts were initiated by the infall of NGC 1404.

2. The sloshing cold front to the northeast at a radius of $10 \mathrm{kpc}$ is the most prominent front, separating the lowentropy high-metallicity gas and the high-entropy lowmetallicity gas.

3. The spatial distribution of enriched ICM traces the spiral morphology of the gas sloshing. In this particular cluster,
Table 2

Fluorescent Instrumental Lines Produced by the Hard Particles

\begin{tabular}{|c|c|c|c|}
\hline Energy (keV) & Line & Energy $(\mathrm{keV})$ & Line \\
\hline \multicolumn{2}{|c|}{ MOS } & \multicolumn{2}{|c|}{ pn } \\
\hline 1.49 & $\mathrm{Al} \mathrm{K}_{\alpha}$ & 1.48 & Al $K_{\alpha}$ \\
\hline 1.75 & Si $\mathrm{K}_{\alpha}$ & 4.51 & Ti K $\alpha$ \\
\hline 5.41 & $\mathrm{Cr} \mathrm{K}{ }_{\alpha}$ & 5.42 & $\mathrm{Cr} \mathrm{K}{ }_{\alpha}$ \\
\hline 5.90 & $\operatorname{Mn~} \mathrm{K}_{\alpha}$ & 6.35 & $\mathrm{Fe} \mathrm{K}_{\alpha}$ \\
\hline 6.40 & $\mathrm{Fe} \mathrm{K}_{\alpha}$ & 7.47 & $\mathrm{Ni} \mathrm{K}_{\alpha}$ \\
\hline 7.48 & $\mathrm{Ni} \mathrm{K}_{\alpha}$ & 8.04 & $\mathrm{Cu} \mathrm{K}{ }_{\alpha}$ \\
\hline 8.64 & $\mathrm{Zn} \mathrm{K} \mathrm{K}_{\alpha}$ & 8.60 & $\mathrm{Zn} \mathrm{K} \alpha$ \\
\hline \multirow[t]{2}{*}{9.71} & $\mathrm{Au} \mathrm{L} \mathrm{L}_{\alpha}$ & 8.90 & $\mathrm{Cu} \mathrm{K} \beta$ \\
\hline & & 9.57 & $\mathrm{Zn} \mathrm{K}$ \\
\hline
\end{tabular}

gas sloshing is more effective at redistributing the enriched gas than is AGN outflow.

4. The innermost sloshing cold front resides within a radius of $3 \mathrm{kpc}$, which is at a smaller radius than the locations of AGN bubbles. A subkiloparsec bright feature consistent with $\mathrm{KHI}$ eddies is visible at the interface, which allows us to rule out a full Spitzer viscosity and put an upper limit of $10 \mu \mathrm{G}$ on the ordered magnetic field. We identify two other such features to the east where the temperature gradient is smaller, implying that KHI accelerates the heat transport. Alternatively, these features are thermally unstable X-ray filaments.

5. The sloshing timescale is $10 \times$ shorter than the cooling time at cluster center. Gas sloshing is a plausible mechanism to alleviate the cooling problem.

We acknowledge helpful discussions with Eugene Churazov. This work was supported by Chandra Awards GO1-12160X and GO2-13125X issued by the Chandra X-ray Observatory Center, which is operated by the Smithsonian Astrophysical Observatory under NASA contract NAS8-03060.

\section{Appendix XMM-Newton Background Modeling}

We consider two sources of background: astrophysical X-ray background (AXB) and NXB. The model of AXB contains a power-law pow $_{C X B}$ with index $\Gamma=1.41$ characterizing the cosmic X-ray background (CXB, De Luca \& Molendi 2004), a thermal emission ape $\mathrm{c}_{\mathrm{MW}}$ with a temperature of $0.2 \mathrm{keV}$ for the Milky Way emission, and another thermal emission apec $_{\mathrm{LB}}$ with a temperature of $0.08 \mathrm{keV}$ for the Local Bubble emission. Metal abundance and redshift were fixed at 1 and 0 , respectively, for apec $_{M W}$ and apec $c_{\mathrm{LB}}$. All the AXB components except ape $\mathrm{C}_{\mathrm{LB}}$ are expected to be absorbed by foreground (Galactic) cooler gas, characterized by the phabs model. The best-fit surface brightnesses of all AXB components are listed in Table 3, determined with a joint-fit of six offset pointings.

The NXB component contains a set of fluorescent instrumental lines and a continuum spectrum for each MOS and pn detector. Fluorescent instrumental lines produced by the hard particles were modeled by a set of Gaussian lines. Their centroid energies are listed in Table 2, taken from Mernier et al. (2015), which were modified from Snowden \& Kuntz (2013). We set an upper limit of $0.3 \mathrm{keV}$ on each line width. We use a power-law model to characterize the continuum particle background component (Snowden \& Kuntz 2013). Even after 
Table 3

X-Ray Background Model Parameters

\begin{tabular}{lcc}
\hline \hline Background & $\mathrm{kT}(\mathrm{keV})$ or $\Gamma$ & norm $\left(\times 10^{-6}\right.$ per $\left.\operatorname{arcmin}^{2}\right)$ \\
\hline CXB & 1.41 & $0.61 \pm 0.07$ \\
MW & 0.2 & $0.44 \pm 0.07$ \\
LB & 0.08 & $1.33 \pm 0.11$ \\
\hline
\end{tabular}

filtering soft flare events from raw data sets, a quiescent level of soft proton flare may remain. To estimate its effect on each $\mathrm{CCD}$, we compared the area-corrected count rates in the 6-12 keV energy band within the field of view (excluding the central $10^{\prime}$ ) and that in the unexposed corners. If this ratio is below 1.15, we consider the event not contaminated by the residual soft proton flare (De Luca \& Molendi 2004). For contaminated events, we add an additional power-law pow SB $_{\text {to }}$ model this residual soft proton component. Its index is allowed to vary between 0.1 and 1.4 (Snowden \& Kuntz 2013). The spectral fit was restricted to the $0.3-10.0 \mathrm{keV}$ energy band.

\section{ORCID iDs}

Paul E. J. Nulsen (1D https://orcid.org/0000-0003-0297-4493

Ralph P. Kraft (ib https://orcid.org/0000-0002-0765-0511

William R. Forman (iD https://orcid.org/0000-0002-9478-1682

Jimmy A. Irwin (1) https://orcid.org/0000-0003-4307-8521

Scott W. Randall ib https://orcid.org/0000-0002-3984-4337

\section{References}

Ascasibar, Y., \& Markevitch, M. 2006, ApJ, 650, 102

Asplund, M., Grevesse, N., \& Jacques, S. A. 2006, NuPhA, 777, 1

Balbus, S. A., \& Soker, N. 1990, ApJ, 357, 353

Bîrzan, L., Rafferty, D. A., McNamara, B. R., et al. 2004, ApJ, 607, 800

Blanton, E. L., Randall, S. W., Clarke, T. E., et al. 2011, ApJ, 737, 99

Buote, D. 2000, MNRAS, 311, 176

Cappellari, M., \& Copin, Y. 2003, MNRAS, 342, 345

Cavagnolo, K., Donahue, M., Voit, M., et al. 2009, ApJS, 182, 12

Chandrasekhar 1961, Hydrodynamic and Hydromagnetic Stability (Oxford: Oxford Univ. Press)

Churazov, E., Forman, W., Jones, C., et al. 2003, ApJ, 590, 225

Churazov, E., Sunyaev, R., Forman, W., et al. 2002, MNRAS, 332, 729

Cox, J. P. 1980, Theory of Stellar Pulsations (Princeton, NJ: Princeton Univ. Press)

David, L. P., Lim, J., Forman, W., et al. 2014, ApJ, 792, 94

David, L. P., \& Nulsen, P. E. J. 2008, ApJ, 689, 837

David, L. P., Vrtilek, J., O’Sullivan, E., et al. 2017, ApJ, 842, 84

De Grandi, S., \& Molendi, S. 2001, ApJ, 551, 153

De Luca, A., \& Molendi, S. 2004, A\&A, 419, 837

Diehl, S., \& Statler, T. S. 2006, MNRAS, 368, 497

Dupke, R., \& White, R. E., III 2000, ApJ, 537, 123

Dursi, L. J., \& Pfrommer, C. 2008, ApJ, 677, 993

Finoguenov, A., \& Jones, C. 2001, ApJ, 547, 107

Fujita, Y., Matsumoto, T., \& Wada, K. 2004, JKAS, 37, 571
Ghizzardi, S., De Grandi, S., \& Molendi, S. 2014, A\&A, 570, 117

Govoni, F., \& Feretti, L. 2004, IJMPD, 13, 1549

Hudson, D. S., Mittal, R., Reiprich, T. H., et al. 2010, A\&A, 513, 37

Ichinohe, Y., Simionescu, A., Werner, N., \& Takahashi, T. 2017, MNRAS, 467,3662

Irwin, J. A., Athey, A. E., \& Bregmanman, J. N. 2003, ApJ, 587, 356

Jones, C., \& Forman, W. 1984, ApJ, 276, 38

Jones, C., Stern, C., Forman, W., et al. 1997, ApJ, 482, 143

Keshet, U., Markevitch, M., Birnboim, Y., \& Loeb, A. 2010, ApJL, 719, L74

Kirkpatrick, C. C., \& McNamara, B. R. 2015, MNRAS, 452, 4361

Komarov, S. V., Churazov, E. M., Schekochihin, A. A., et al. 2014, MNRAS, 440,1153

Lamb, H. 1932, Hydrodynamics (6th ed.; Cambridge: Cambridge Univ. Press) Lyutikov, M. 2006, MNRAS, 373, 73

Machacek, M., Dosaj, A., Forman, W., et al. 2005, ApJ, 621, 663

Markevitch, M., Mazzotta, P., Vikhlinin, A., et al. 2003, ApJ, 586, 19L

Markevitch, M., \& Vikhlinin, A. 2007, PhR, 443, 1

McCourt, M., Sharma, P., Quataert, E., et al. 2012, MNRAS, 419, 3319

Mernier, F., de Plaa, J., Lovisari, L., et al. 2015, A\&A, 575, 37

Morsony, B. J., Heinz, S., Brüggen, M., et al. 2010, MNRAS, 407, 1277

Murakami, H., Komiyama, M., Matsushita, K., et al. 2011, PASJ, 63, S963

Navarro, J. F., Frenk, C. S., \& White, S. D. M. 1997, ApJ, 490, 493

Nulsen, P. E. J., David, L. P., McNamara, B. R., et al. 2002, ApJ, 568, 163

Paolillo, M., Fabbiano, G., Peres, G., \& Kim, D.-W. 2002, ApJ, 565, 883

Peterson, J. R., \& Fabian, A. C. 2006, PhR, 427, 1

Rangarajan, F. V. N., Fabian, A. C., Forman, W. R., et al. 1995, MNRAS, 272, 665

Rebusco, P., Churazov, E., Böhringer, H., et al. 2005, MNRAS, 359, 1041

Roediger, E., Brüggen, M., Simionescu, A., et al. 2011, MNRAS, 413, 2057

Roediger, E., Kraft, R. P., Machacek, M. E., et al. 2012a, MNRAS, 754, 147

Roediger, E., Kraft, R. P., Nulsen, P. E. J., et al. 2013, MNRAS, 436, 1721

Roediger, E., Kraft, R. P., Nulsen, P. E. J., et al. 2015a, ApJ, 806, 103

Roediger, E., Kraft, R. P., Nulsen, P. E. J., et al. 2015b, ApJ, 806, 104

Roediger, E., Lovisari, L., Dupke, R., et al. 2012b, MNRAS, 420, 3632

Rossetti, M., \& Molendi, S. 2010, A\&A, 510, A83

Sanders, J. S., Fabian, A. C., Russell, H. R., et al. 2016a, MNRAS, 460, 1898

Sanders, J. S., Fabian, A. C., Taylor, G. B., et al. 2016b, MNRAS, 457, 826

Sanderson, A., O'Sullivan, E., \& Ponman, T. 2009, MNRAS, 395, 764

Sarazin, C. 1988, Cambridge Astrophysics Series (Cambridge: Cambridge Univ. Press)

Scharf, C. A., Zurek, D. R., \& Bureau, M. 2005, ApJ, 633, 154

Simionescu, A., Werner, N., Böhringer, H., et al. 2009, A\&A, 493, 409

Skrutskie, M. F., Cutri, R. M., Stiening, R., et al. 2006, AJ, 131, 1163

Snowden, S. L., \& Kuntz, K. D. 2013, XMM ESAS cookbook

Spitzer 1956, Physics of Fully Ionized Gases (New York: Interscience)

Su, Y., Buote, D., Gastaldello, F., et al. 2016, ApJ, 821, 40

Su, Y., Kraft, R. P., Nulsen, P. Ė. J., et al. 2017a, ApJ, 835, 19

Su, Y., Nulsen, P. Ė. J., Kraft, R. P., et al. 2017b, ApJ, 847, 94 (Paper 1)

Su, Y., Kraft, R. P., Roediger, E., et al. 2017c, ApJ, 834, 74

Vaddi, S., O'Dea, C. P., Baum, S. A., et al. 2016, ApJ, 818, 182

Vikhlinin, A., Markevitch, M., \& Murray, S. S. 2001, ApJ, 551, 160

Voit, G. M., Donahue, M., Bryan, G. L., et al. 2015, Natur, 519, 203

Walker, S. A., Hlavacek-Larrondo, J., Gendron-Marsolais, M., et al. 2017, MNRAS, 468, 2506

Walker, S. A., Sanders, J. S., Fabian, A. C., et al. 2016, MNRAS, 461, 684

Zhuravleva, I., Allen, S. W., Mantz, A. B., et al. arXiv:1707.02304

ZuHone, J. A., Kunz, M. W., Markevitch, M., et al. 2015, ApJ, 798, 90

ZuHone, J. A., Markevitch, M., \& Johnson, R. 2010, ApJ, 717, 908

ZuHone, J. A., Markevitch, M., \& Lee, D. 2011, ApJ, 743, 16

ZuHone, J. A., Markevitch, M., Ruszkowski, M., \& Lee, D. 2013, ApJ, 762, 69 